\title{
Tracking Formation Control for Multiple Quadrotors Based on Fuzzy Logic Controller and Least Square Oriented by Genetic Algorithm
}

\author{
Rabah Abbas* and Qinghe Wu
}

School of Automation, Beijing Institute of Technology, Beijing 100081, Beijing, China

\begin{abstract}
This paper studies the leader-follower formation control for multiple quadrotors. Two controllers are used. The first one is a proportional derivative controller used to ensure the tracking of the leader to the desired trajectory, while the second is based on fuzzy logic in order to achieve the desired formation in $X-Y$ plane with equal height ( $z$ ) for all follower quadrotors. In order to ensure the speed time convergence of the formation shape, Genetic Algorithm is used online to tune the fuzzy logic controller parameters. This genetic algorithm is also used to predict the trajectory of the quadrotor leader in the case of communication failure between leader and follower quadrotors by online estimation of the least square coefficients. Proportional derivative controller is used again to keep the desired formation shape of the follower quadrotors. Finally, simulation results demonstrate the effectiveness of the proposed algorithms.
\end{abstract}

Keywords: Fuzzy Logic Controller (FLC), Genetic Algorithm (GA), Proportional Derivative (PD), Quadrotor, Leader Follower, Unmanned Aerial Vehicle (UAV).

\section{INTRODUCTION}

Formation control of multi-agent systems has become the interest of many researches in the world $[1,2]$. It presents many advantages such as robustness, lower cost, and the increasing of probability success [3, 4]. In the literature different architectures and strategies have been proposed, such as behavior-based, virtual structure, potential field and leader follower.

In this paper we propose an algorithm based on leader follower architecture. This technique is the most popular due to its simplicity in implementation [5]. Due to the different advantages of quadrotors, tracking formation control for multiple quadrotors has become the interest of many researches in the world. In [6], the sliding mode controller is used to guide the law for the heading control of the leader unmanned aerial vehicle (UAV), while for each follower, heading rate controller is developed to achieve a circular formation around the leader. In [7], the leader UAV is controlled to follow a predefined path by using waypoints, then based on Linear Quadratic Regulator (LQR) and neural control the follower UAVs are commanded to fly with a threedimensional offset with respect to the leader. In [8], based on the bounded input, the tracking of small quadrotors is studied. In [9], the formation of UAVs is achieved by using the measurement of the inertial position of each follower and also the position of leader. In [10] based on linear proportional derivative and sliding mode controller, flight formation control for leader follower quadrotor is presented, it is tested in real application and it shown a good results.

*Address correspondence to this author at the School of Automation, Beijing Institute of Technology, Beijing 100081, Beijing, China;

E-mail: abbas_rabah@hotmail.fr
Based on the work [10], in this study, $l-\emptyset$ control is proposed to ensure the formation of multiple quadrotors. Two controllers are used. First, PD controller is used to ensure the tracking of the leader to the desired trajectory. PD controller provides high sensitivity and tends to increase the stability of the system. The second controller is based on fuzzy logic. It is used in order to achieve the desired formation in $X-Y$ plane with equal height (z) for all follower quadrotors. FLC shows a good result in tracking control of robots [11], helicopters $[12,13]$. FLC has many advantages such as simplicity of control, low cost and the possibility to design of controller without the exact knowledge of the mathematical model of the controlled system. Finally, PD controller is used again to keep the desired formation shape of the follower quadrotors.

The success of the FLC controller to achieve a formation shape of the team of quadrotors in a possible short time depends on an adequate choice of the FLC parameters. For this reason we use the genetic algorithm to tune online these parameters for each follower. GA presents many advantages over other mathematical programming techniques [14]. GA is a robust optimization technique because it ensure a gradual increasing of a good solution and it has lower chance to converge into local minima.

In the leader follower approach, if the communication between the leader and the follower is failed, the formation cannot be maintained and consequently the quadrotors will be crashed which is not taken into account in our previous work [15]. To overcome to this problem, many prediction techniques are presented in literature such as artificial neural networks (ANN), linear and nonlinear least square techniques. ANN provides a good result in real applications [16, 17], but it is more complex due to the network structure which generates more time in running. One good and 


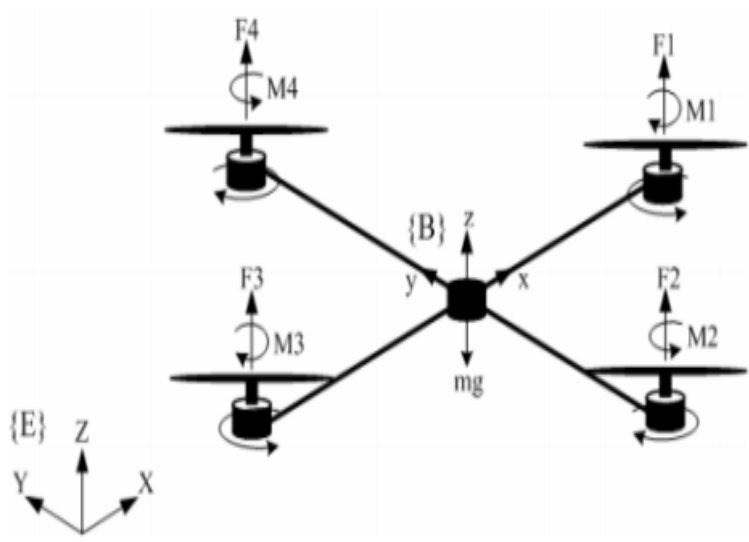

Fig. (1). Quadrotor configuration.

suitable solution in our case is the use of GA to estimate the least square parameters. Using GA with small population size and repeating experiments many times reduces the run time compared to the ANN which is suitable in real time application. In [18, 19], authors estimate the least square parameters by finding the smallest fitness function, after that the corresponding parameters will be selected. Satoshi et al. used the multiple GA to estimate the least square parameters; authors focus on the initial (minimal and maximal) of each parameter different to another, and finally the algorithm select the best parameters according to the best fitness function of multiple GA used in the algorithm [20]. In our study and in order to maintain the formation shape between quadrotors even the communication between the leader and followers is failed, we propose an algorithm based on GA to estimate online the least square parameters by minimizing the sums of squares of residuals between the estimated function and experimental data.

\section{QUADROTOR DYNAMIC MODEL}

In this paper, we consider the model dynamic of quadrotor Fig. (1) based on Newton-Euler approach.

The dynamic model is presented as in [21]:

$$
\left\{\begin{aligned}
\ddot{x} & =u_{1}(\cos \varphi \sin \theta \cos \psi+\sin \varphi \sin \psi) \\
\ddot{y} & =u_{1}(\cos \varphi \sin \theta \sin \psi-\sin \varphi \cos \psi) \\
\ddot{z} & =u_{1}(\cos \varphi \cos \theta)-g \\
\ddot{\varphi} & =u_{2} l \\
\ddot{\theta} & =u_{3} l \\
\ddot{\psi} & =u_{4}
\end{aligned}\right.
$$

$(x, y, z)^{T}$ Corresponds to the relative position of the mass centre of the quadrotor with respect to an inertial coordinate frame.

$g$ is the gravitational acceleration. $l$ is the length from the mass centre to the rotor, $(\varphi, \theta, \psi)^{\mathrm{T}}$ denotes the three Euler angles that represent the attitude of the quadrotor, namely roll-pitch-yaw of the quadrotor.

These angles are limited by:

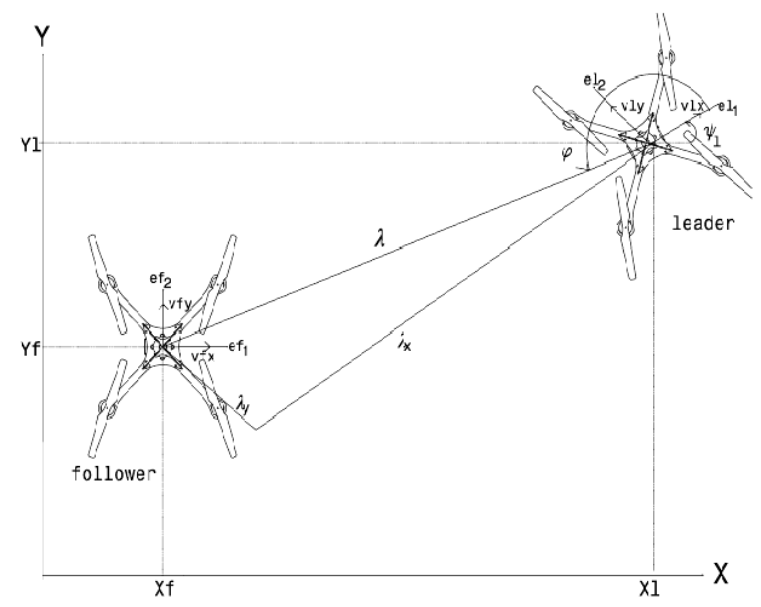

Fig. (2). Position and orientation of the leader and follower quadrotors in $(x-y)$ plane [10].

$-\frac{\pi}{2}<\varphi<\frac{\pi}{2},-\frac{\pi}{2}<\theta<\frac{\pi}{2}$ and $-\pi<\psi<\pi$.

$u_{1}$ is the thrust force vector in the body system, $u_{2}, u_{3}$ and $u_{4}$ correspond to the control inputs of roll, pitch and yaw moments, respectively.

\section{CONTROLLER DESIGN}

In this section, two controllers are designed to ensure the tracking and the formation for multiple quadrotors, respectively.

\subsection{Tracking Controller}

A simple PD is used to ensure the tracking of the desired trajectory by the first quadrotor designated as leader. This controller will be used again to ensure the keeping of formation in $x-y$ plane. The controller can be expressed as:

$u_{i}=K_{p} e_{i}+K_{D} \dot{e}_{i}$

With $e_{i}$ and $\dot{e}_{i}$ are the error and the error derivation in $i$ th direction. $K_{p}$ and $K_{D}$ are the propositional and derivative gains, respectively.

\subsection{Formation Controller Design}

The formation control is ensured by keeping a fixed distance $D$ and a fixed deviation $\Delta \psi$ between the leader and the $i$-th follower quadrotor (Fig. 2).

Now, considering $N$ quadrotors. In our study we consider that the quadrotors have the same translational dynamic model in $X-Y$ plane. It is given by the following system [10]:

$$
\left\{\begin{array}{l}
\dot{x}_{i}=v_{i x} \cos \left(\psi_{i}\right)-v_{i y} \sin \left(\psi_{i}\right) \\
\dot{y}_{i}=v_{i x} \sin \left(\psi_{i}\right)+v_{i y} \cos \left(\psi_{i}\right) \\
\dot{\psi}_{i}=\omega_{i}
\end{array}\right.
$$




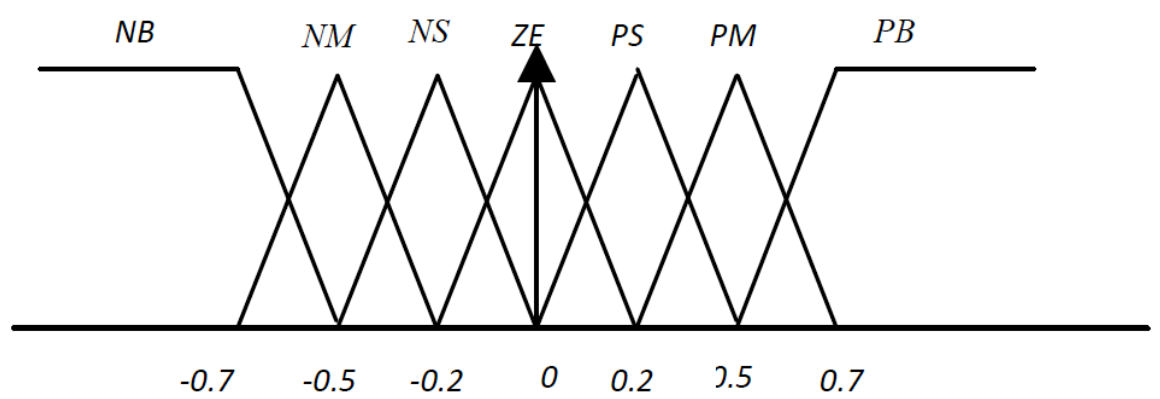

Fig. (3). Membership function for $\psi$ and $\Delta \psi$.

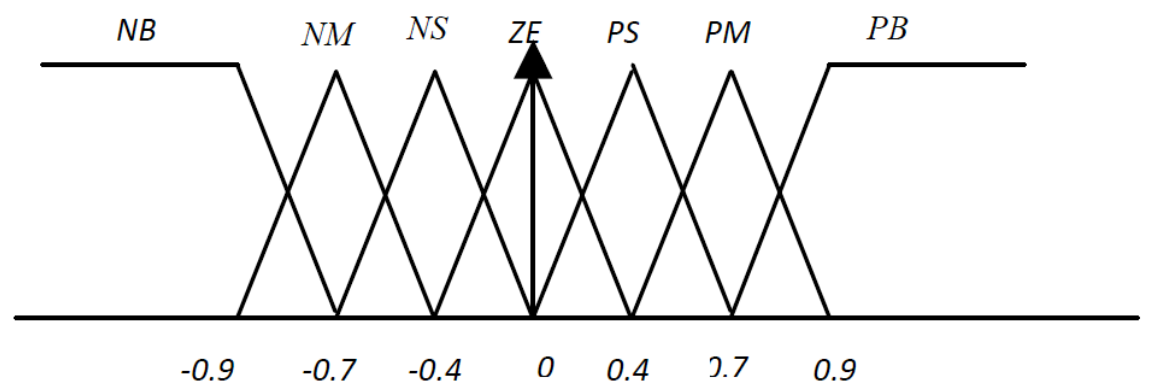

Fig. (4). Membership function for $E D$.

Where: $v_{i x}$ and $v_{i y}$ are the velocity component in $x$ and $y$ directions, respectively.

$\omega_{i}$ is the angular velocity for the yaw angle $\psi$.

\subsubsection{Distance and Angle Controller}

The distance controller is used to compute the two linear velocities $v_{x}$ and $v_{y}$ in $x$ and $y$ direction, respectively. So, we can write :

$v_{n}=K_{n} F L C\left(E D_{n}\right)$

With $K_{n}$ is positive constant, and $n$ represents $x$ or $y$ direction.

The angle controller is used to calculate the angular velocity $(w)$ for the yaw angle in order to maintain a fixed deviation angle between the leader and follower quadrotors. So we can write:

$w=K_{w} F L C(\Delta \psi, E \psi)$

With $K_{w}$ is positive constant.

The distance and the angle deviation between the leader and the follower quadrotors (Fig. 2) can be expressed as:

$\left\{\begin{aligned} D & =\sqrt{D_{x}{ }^{2}+D_{y}{ }^{2}} \\ D x & =x_{l}-x_{f} \\ D y & =y_{l}-y_{f} \\ \Delta \psi & =\psi_{l}-\psi_{f}\end{aligned}\right.$

The distance and angle errors between leader and follower quadrotor are given by:
$\left\{\begin{array}{c}E D=D_{\text {des }}-D \\ E \psi=\Delta \psi_{\text {des }}-\Delta \psi\end{array}\right.$

Where: $D_{\text {des }}, \Delta \psi_{\text {des }}$ are the desired distance and angle separation between the leader and follower quadrotors, respectively.

The position and angular errors are represented by seven linguistic fuzzy sets (NB Negative Big, NM Negative Medium, NS Negative Small, ZE Zero, PS Positive Small, PM Positive Medium and PB Positive Big), with the membership shown in the Fig. (3) and Fig. (4), respectively.

The two velocity components in the $x$ and $y$ directions $\left(v_{i x}\right.$ and $\left.v_{i y}\right)$ are considered as the control variables of the quadrotor. They are also presented by seven linguistic fuzzy sets (NB,NM,NS,ZE,PS,PM,PB), with the membership shown in the Fig. (5)

The defuzzification strategy is implemented by the weighted average method. The output of the controllers can be given by:

$u=\frac{\sum_{i=1}^{N} \mu_{i}\left(u_{i}\right) u_{i}}{\sum_{i=1}^{N} \mu_{i}\left(u_{i}\right)}$

$u$ can present one of the two linear velocities $v_{x}$ or $v_{y}$ and can also presents the angular velocity $w$.

$\mu_{i}$ is the support of each fuzzy set. $\mu_{i}\left(u_{i}\right)$ is the membership function value of each rule $i$ and $\mathrm{N}$ is the number of control, $\mu_{i}$ is the level activation of rule $i \mathrm{j}$.

$N=7$ for the linear velocities control (Table 1).

$N=49$ for the angular velocity control (Table 2 ). 


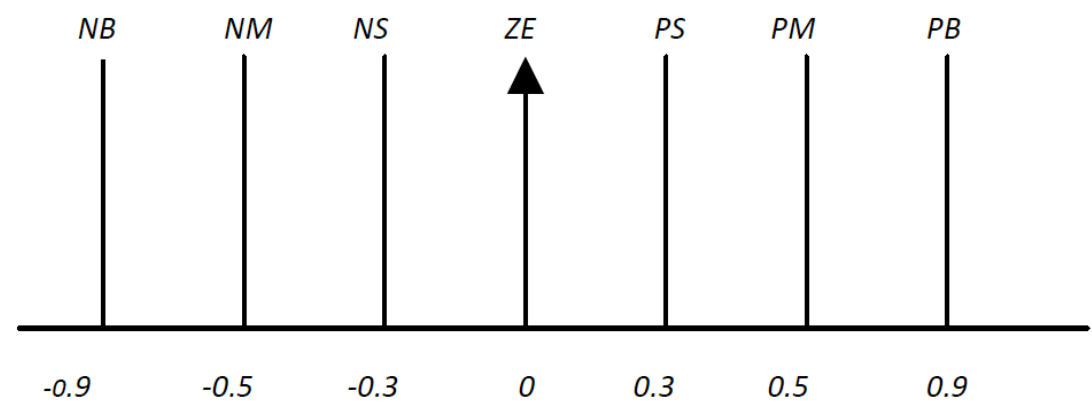

Fig. (5). Membership function for $v_{x}, v_{y}$ and $w$.

\subsection{Optimization}

In this section, we use the genetic algorithm to tune online the FLC parameters $\left(K_{x}, K_{y}, K_{w}\right)$ for each follower.

\subsubsection{Genetic Algorithm GA}

After the fixation of the range variation of scaling factor (minimal and maximal values), an initial random population of individuals will be generated, then a fitness function for each chromosome is calculated. The reproduction is reserved only for the fittest individuals. After that, it breeds new individuals through different genetic operations of crossover and mutation. The operations will be repeated until the achieving the convergence criterion.

\subsubsection{Optimization}

In this section, we interested to tune the FLC parameters $\left(K_{x}, K_{y}, K_{\psi}\right)$ by using GA. For better time convergence of the formation control errors, GA is online used.

In this case, we defined the fitness function $J_{1}$ as:

$J_{1}=e_{D}+\Delta e_{\psi}$.

With: $e_{D}=D^{d}-\sqrt{D_{x}^{2}+D_{y}^{2}}$ and $\Delta e_{\psi}=e \psi^{d}-e \psi$ are the distance and the orientation errors between the leader and follower quadrotor, respectively.

In this algorithm and different to the static technique, for each iteration of algorithm, a best function is selected and a best controller parameters correspond to this function are also selected and online injected in the controller (04) and (05). The same procedure will be repeated until the $n$-th iteration.

\subsubsection{Estimation of Leader Trajectory Using Least Square}

In this section and in order to overcome to the communication failure problem, we propose an algorithm based on GA in order to estimate online the least square parameters.

Let the estimated nonlinear function $\hat{Y}$ takes the following form:

$\hat{Y}=a_{0}+a_{1} y+a_{2} y^{2}+\cdots+a_{n} y^{n}$

with $a_{i}$ are the coefficients will be estimated.
In order to estimate the different parameters, we use GA as described in section (3.3.1) by choosing the fitness function as the sum of nonlinear least square:

$J_{2}=\frac{1}{2}\left(\sum_{i=1}^{n}\left(Y-a_{i} y^{i}\right)^{2}\right)$

\section{SIMULATION}

The proposed formation control has been simulated for the case of three quadrotors (one leader and two followers).

The controllers' objectives are:

First: The desired trajectory tracked by the leader is described by:

$x_{d l}=t, y_{d l}=\cos (\mathrm{t}), z_{d l}=5 \mathrm{~m}$ and $\psi_{d l}=0$.

Second: The formation and the keeping of formation by the followers described by the desired distance and deviation angle to the leader:

Follower $\quad 1:\left\{\begin{array}{l}x_{l}-x_{f 1}=2 \\ y_{l}-y_{f 1}=-1\end{array} \quad, \quad D_{d 12}=\sqrt{5} \quad\right.$ meters and $\Delta \psi_{\text {des } 12}=0$

Follower 2: $\left\{\begin{array}{l}x_{l}-x_{f 2}=2 \\ y_{l}-y_{f 2}=1\end{array} \quad, \quad D_{d 13}=\sqrt{5} \quad\right.$ meters and $\Delta \psi_{\operatorname{des} 13}=0$.

The controller parameters for the first and second followers are taken as:

$K_{x 1}=5, K_{y 1}=8, K_{w 1}=0.09$

$K_{x 2}=5, K_{y 2}=8, K_{w 2}=0.09$

The length from the mass centre to the rotor $l$ is taken $l=50 \mathrm{~cm}$ and the gravitational accelearation $g=9.81 \mathrm{~m} /$ $s^{2}$.

The different simulations are depicted from Fig. (6) to Fig. (15).

Fig. (6) depicts the trajectories of the position $z(t)$ for the quadrotors. The trajectories of the leader and follower quadrotors in $(x-y)$ plane (Desired formation) are depicted in Fig. (7), while the distance and the angle errors between 
Table 1. Rule table of distance controller.

\begin{tabular}{|c|c|c|c|c|c|c|c|}
\hline EDx, EDy & NB & NM & NS & ZE & PS & PM & PB \\
\hline \hline$V x, V y$ & $N B$ & $N M$ & $N S$ & $Z E$ & $P S$ & $P M$ & $P B$ \\
\hline
\end{tabular}

Table 2. Rule table of angle controller.

\begin{tabular}{|c|c|c|c|c|c|c|c|}
\hline & NB & NS & NM & $\mathbf{Z E}$ & PS & PM & PB \\
\hline NB & $\mathrm{PB}$ & PB & $\mathrm{PB}$ & PB & PB & PS & ZE \\
\hline NS & PB & PB & $\mathrm{PM}$ & PM & PS & ZE & NS \\
\hline NM & $\mathrm{PB}$ & PM & PS & PS & ZE & NS & PB \\
\hline $\mathrm{ZE}$ & PB & $\mathrm{PM}$ & PS & $\mathrm{ZE}$ & NS & NM & $\mathrm{NB}$ \\
\hline PS & PB & PS & $\mathrm{ZE}$ & NS & NS & NM & NB \\
\hline PM & PS & $\mathrm{ZE}$ & NS & NM & NM & NB & $\mathrm{NB}$ \\
\hline PB & $\mathrm{ZE}$ & NS & NB & NB & NB & NB & NB \\
\hline
\end{tabular}

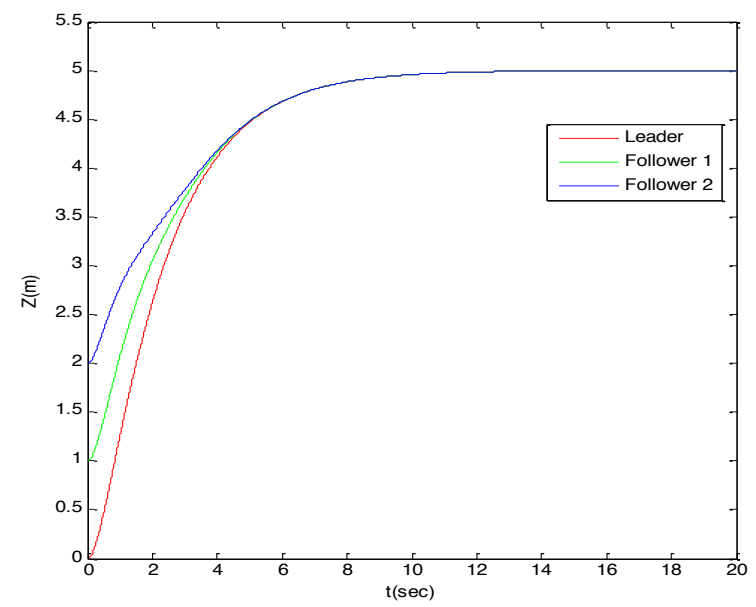

Fig. (6). Trajectories of leader and follower quadrotors in $z$ direction.

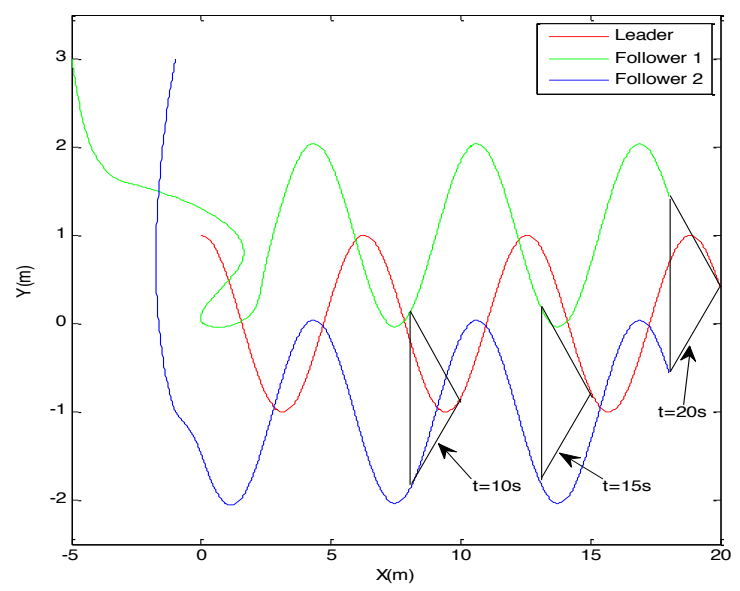

Fig. (7). Trajectories of leader and follower quadrotors in $x-y$ plane before optimization. 


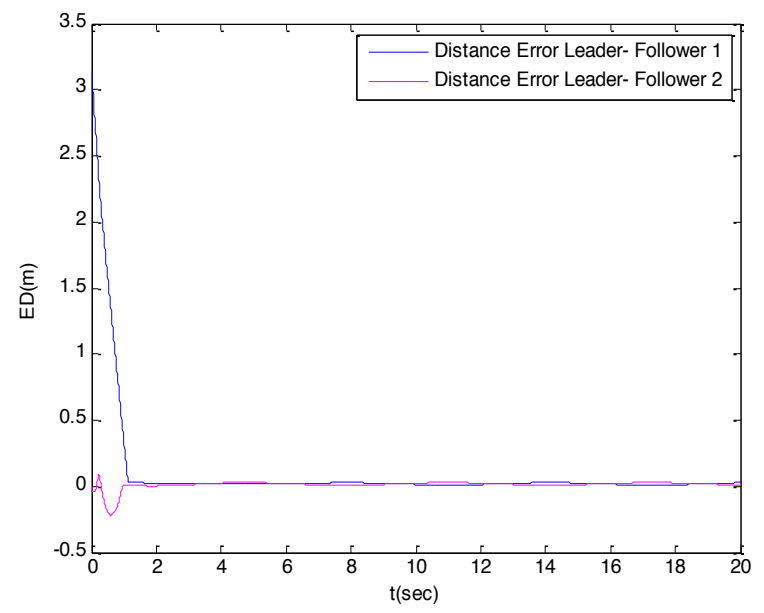

Fig. (8). Distance error between leader and follower quadrotors before optimization.

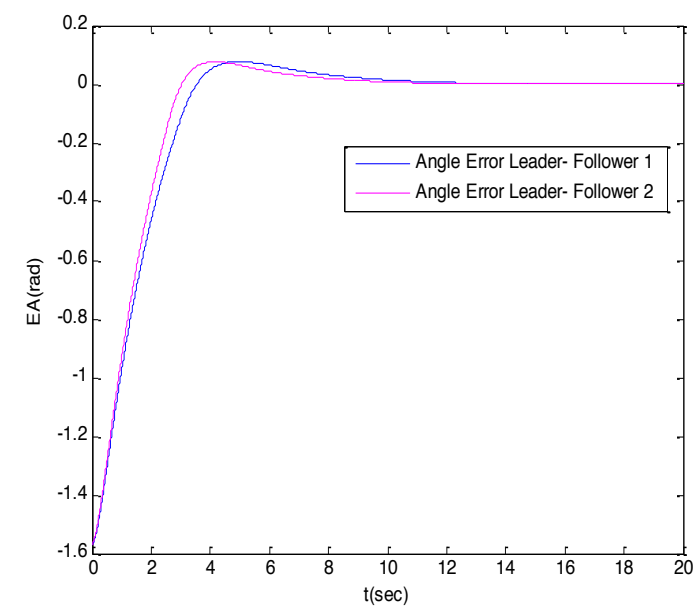

Fig. (9). Angle error between leader and follower quadrotors before optimization.

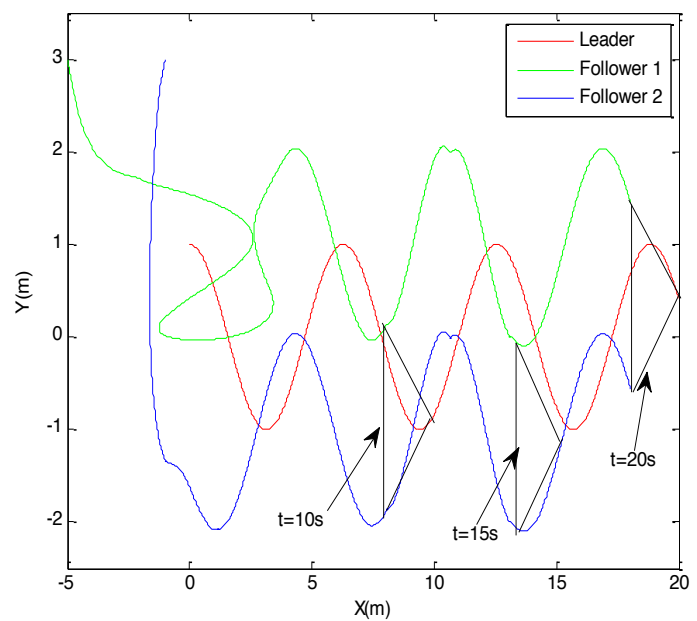

Fig. (10). Trajectories of leader and follower quadrotors in $x-y$ plane by offline optimization. 


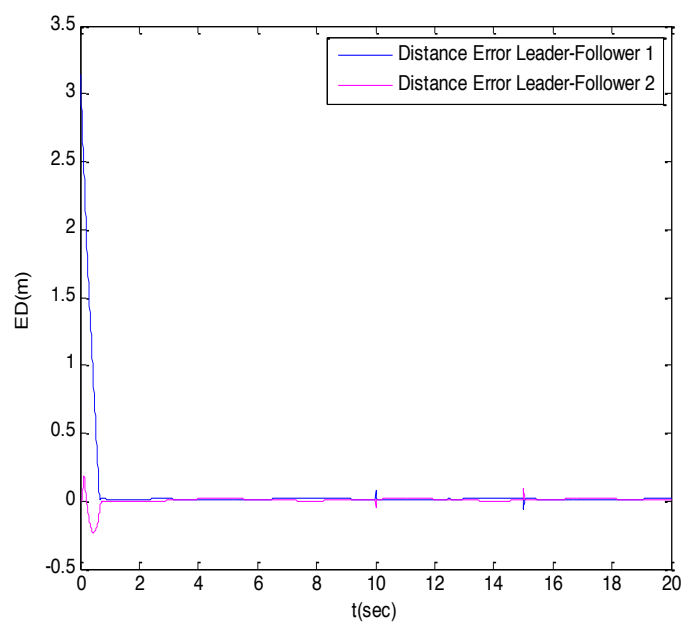

Fig. (11). Distance error between leader and follower quadrotors by using offline optimization.

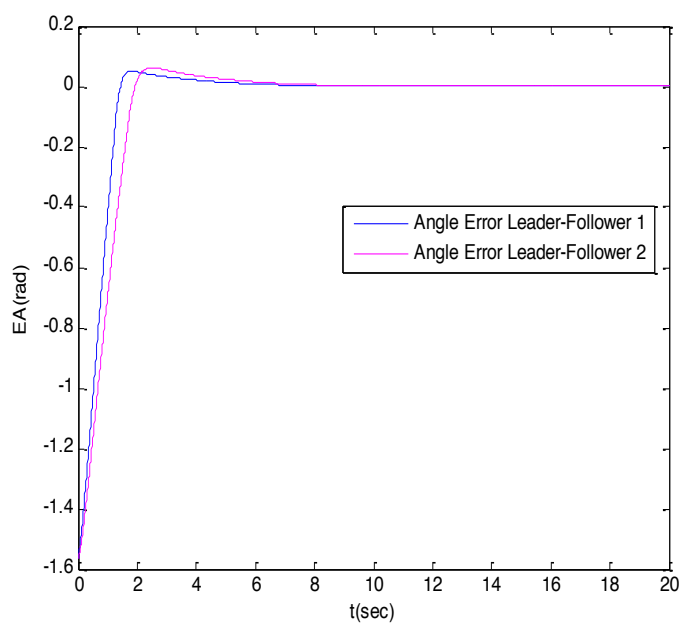

Fig. (12). Angle error between leader and follower quadrotors by using offline optimization.

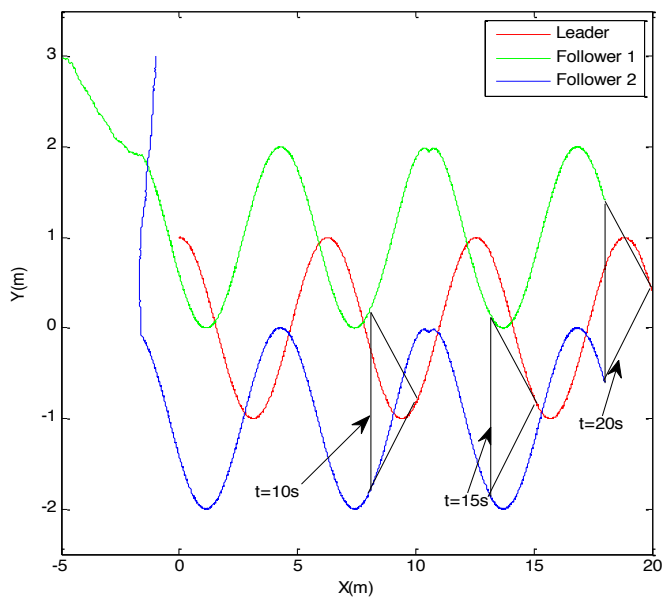

Fig. (13). Trajectories of leader and follower quadrotors in $x-y$ plane by online optimization. 


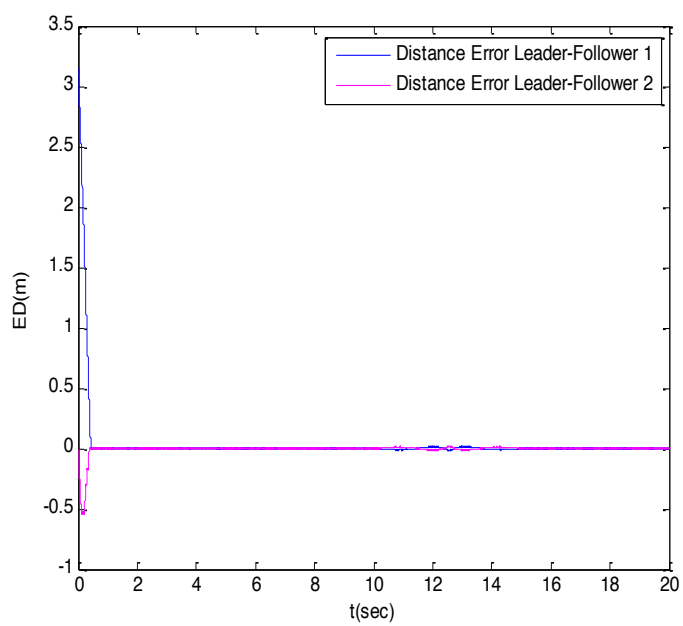

Fig. (14). Distance error between leader and follower quadrotors by using online optimization.

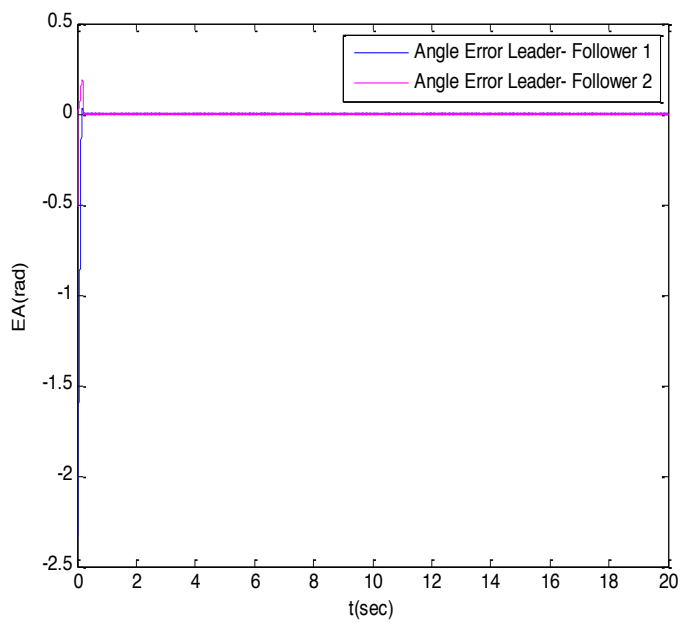

Fig. (15). Angle error between leader and follower quadrotors by using online optimization.

leader and follower quadrotors by using the fuzzy logic controller are depicted in Fig. (8) and Fig. (9), respectively.

By applying GA, the minimal fitness function is equal to $J_{1}=0.98$

And the FLC parameters corresponding are given by:

Follower1: $K_{x 1}=5.68, K_{y 1}=7.24, K_{w 1}=0.53$

Follower2: $K_{x 1}=6.29, K_{y 1}=4.59, K_{w 1}=0.53$

In online case, the fitness function and the corresponding parameters are variables.

Fig. (13), Fig. (14) and Fig. (15) show a good performances in terms of convergence speed of the tracking errors and time achieving of the desired formation by tuning online the FLC parameters compared to the offline case which is depicted from Fig. (13) to Fig. (15).

Now, we interrupt the communication between the leader and follower quadrotors between $\mathrm{t}=10$ and $\mathrm{t}=15 \mathrm{~seconds}$.

In offline case, the parameters founded in $\mathrm{x}$-direction are given by: $a_{0}=-0.0384, a_{1}=1.0087$ and the corresponding fitness function is equal to $J_{2 x}=0.0258$.

In $y$-direction, the parameters are given by:

$a_{0}=0.90, a_{1}=0.38, a_{2}=-1.02, a_{3}=0.2$, and the corresponding fitness function is equal to $J_{2 y}=3.48$

In online prediction, the formation shape is achieved very quickly and the parameters are variables and the fitness functions are also variables for each iteration which ensure the best result in term of fitness function compared to the online prediction case. 


\section{CONCLUSION}

This paper addressed the problem of leader follower formation tracking control for multiple quadrotors. An effective algorithm based on fuzzy logic and proportional derivative controllers oriented by genetic algorithm is proposed.

The proposed algorithms present two advantages, first, the speed of convergence of the tracking errors and time achieving of the desired formation by online tuning the fuzzy logic controller parameters. Second, the proposed algorithm can overcome to the communication failure between the leader and the follower quadrotors by online estimation of least square parameters, which is very interesting in real applications.

With the controller designed in this study, a collision might occur between quadrotors. Our future works will include the design of a potential function which will be added in the controller then we will attempt to implement the different algorithms in real application.

\section{CONFLICT OF INTEREST}

The authors confirm that this article content has no conflict of interest.

\section{ACKNOWLEDGEMENTS}

Declared none.

\section{REFERENCES}

[1] J.A. Fax, and R. Murray, "Information flow and cooperative control of vehicle formations", IEEE Transactions on Automatic Control, vol. 49, no. 9, pp. 1453-1464, 2004.

[2] W. Ren, R. W. Beard, and E. M. Atkins, "A survey of consensus problems in multi-agent coordination", In: American Control Conference, Portland, USA, 2005, pp. 1859-1864.

[3] T. Lee, K. Sreenath, and V Kumar, "Geometric control of cooperating multiple quadrotor UAVs with a suspended payload", In: $52^{\text {nd }}$ IEEE Conference on Decision and Control: Italy, 2013, pp. 5510-5515.

[4] M. F. B. Abas, D. Pebrianti, S. A. M. Ali, D. Lwakura, Y. Song, K. Nonami, and D. Fujiwara, "Circular leader-follower, formation control of quad-rotor aerial vehicles", Journal of Robotics and Mechatronics, vol. 25, no. 1, pp. 60-71, 2013.

[5] J. Lee, H. Seok, and Y. kim. "Formation geometry center based formation controller design using Lyapunov stability theorem", International Journal of Aeronautical and Space Sciences. vol. 9, no. 2. pp. 71-78, 2008.
[6] S. Zhu, D. Wang, and C. B. Low, "Cooperative control of multiple UAVs for source seeking", Journal of Inteligent and Robotic Systems, vol. 70, no. 4, pp. 293-301, 2013.

[7] F. Rinaldi, S. Chiesa, and F. Quagliotti, "Linear quadratic control for quadrotors UAVs dynamics and formation flight", Journal of Intelligent \& Robotic Systems, vol. 70, no. 4, pp. 203-220, 2013.

[8] J. A. Guerrero, P. Castillo, S. Salazar, and R. Lozano, "Mini rotorcraft flight formation control using bounded inputs", Journal of Intelligent \& Robotic Systems, vol. 65, no. 4, pp. 175-186, 2012.

[9] B. Yun, B. M. Chen, K. Y. Lum, and T. H. Lee, "Design and implementation of a leader-follower cooperative control system for unmanned helicopters", Journal of Control Theory and Applications, vol. 8, no. 1, pp. 61-68, 2010.

[10] A. Mercado, R. Castro, and R. Lozano, "Quadrotors flight formation control using a leader-follower approach", In: European Control Conference, Zurich, 2013, pp. 3858-3863.

[11] K. Benbouabdallah, and Z. Qi-dan. "Genetic fuzzy logic control technique for a mobile robot tracking a moving target", International Journal of Computer Science Issues, vol. 10, no. 1, pp. 607613, 2013.

[12] S. Zeghlache, D. Saigaa, K. Kara, A. Harrag, and A. Bouguerra. "Fuzzy sliding mode control with chattering elimination for a quadrotor helicopter in vertical flight", Hybrid Artificial Intelligent Systems, vol. 720, no. 8, pp. 125-136, 2012.

[13] D. Gautam, and C. Ha, "Control of a quadrotor using a smart selftuning fuzzy pid controller", International Journal of Advanced Robotic Systems, vol. 10, no. 380, pp. 1-9, 2013.

[14] A. R. Simpson. G. C. Dandy, and L. J. Murphey, "Genetics algorithms compared to other techniques for pipe optimization". Journal of Water Resources Planning and Management, vol. 120, no. 4, 1994.

[15] R. Abbas, and $Q . W u$, "Improved leader follower formation control for multiple quadrotors based AFSA", Telkomnika, Telecommunication, Computing, Electronics and Control, vol. 13, no. 1, 2015.

[16] C. Cheng, K. Chau, Y. Sun, and J. Lin, Long-Term Prediction of Discharges in Manwan Reservoir Using Artificial Neural Network Models, In: Advances in Neural Networks-ISNN, 2005, pp. 10401045 .

[17] R. Taormina, K. W. Chau, and R. Sethi, "Artificial neural network simulation of hourly groundwater levels in a coastal aquifer system of the Venice lagoon", Engineering Applications of Artificial Intelligence, vol. 25, no. 8, pp. 1670-1676, 2012.

[18] S. Senturk, Applied Genetic Algorithms Approach to Curve Fitting Problems, Master's Thesis. The Graduate School of Natural and Applied Sciences, 2009.

[19] C. L. Karr, D. A. Stanley, and B. J. Scheiner, Genetic Algorithm Applied to Least Squares Curve Fitting, U.S. Bureau of Mines Report of Investigations, 1991, p. 9339.

[20] S. Tomioka, S. Nisiyama, and T. Enoto, "Nonlinear least square regression by adaptive domain method with multiple genetic algorithms", IEEE Transactions on Evolutionary Computation, vol. 1, no.1, pp. 1-16, 2007.

[21] D. Lee, H. J. Kim, and S. Sastry. "Feedback linearization vs adaptive sliding mode control for a quadrotor Helicopter", International Journal of Control, Automation and Systems, vol. 7, no.3, pp. 419428. 2009.

(C) Abbas and $\mathrm{Wu}$; Licensee Bentham Open.

This is an open access article licensed under the terms of the Creative Commons Attribution Non-Commercial License (http://creativecommons.org/licenses/by-nc/3.0/) which permits unrestricted, non-commercial use, distribution and reproduction in any medium, provided the work is properly cited. 